\title{
Post-transplant recurrence of steroid resistant nephrotic syndrome in children: the Italian experience
}

\author{
William Morello ${ }^{1}$. Sairaj Puvinathan ${ }^{1}$ - Giuseppe Puccio ${ }^{1}$. Gian Marco Ghiggeri ${ }^{2}$ - Luca Dello Strologo ${ }^{3}$. \\ Licia Peruzzi ${ }^{4}$. Luisa Murer ${ }^{5}$. Michela Cioni ${ }^{2}$. Isabella Guzzo ${ }^{3}$. Enrico Cocchi ${ }^{4}$. Elisa Benetti ${ }^{5}$. Sara Testa ${ }^{1}$. \\ Luciana Ghio ${ }^{1}$. Gianluca Caridi ${ }^{2} \cdot$ Massimo Cardillo $^{6} \cdot$ Rosanna Torelli $^{6} \cdot$ Giovanni Montini ${ }^{1}[0$
}

Received: 29 July 2019 / Accepted: 9 October 2019 / Published online: 15 October 2019

(c) The Author(s) 2019

\begin{abstract}
Background Steroid resistant nephrotic syndrome (SRNS) is a frequent cause of end stage renal disease in children and post-transplant disease recurrence is a major cause of graft loss.

Methods We identified all children with SRNS who underwent renal transplantation in Italy, between 2005 and 2017. Data were retrospectively collected for the presence of a causative gene mutation, sex, histology, duration of pre-transplant dialysis, age at onset and transplant, HLA matching, recurrence, therapy for recurrence, and graft survival.

Results 101 patients underwent a first and 22 a second renal transplant. After a median follow-up of 58.5 months, the disease recurred on the first renal transplant in $53.3 \%$ of patients with a non-genetic and none with a genetic SRNS. Age at transplant $>9$ years and the presence of at least one HLA-AB match were independent risk factors for recurrence. Duration of dialysis was longer in children with relapse, but did not reach statistical significance. Overall, 24\% of patients lost the first graft, with recurrence representing the commonest cause. Among 22 patients who underwent a second transplant, 5 suffered of SRNS recurrence. SRNS relapsed in 5/9 (55\%) patients with disease recurrence in their first transplant and 2 of them lost the second graft.

Conclusions Absence of a causative mutation represents the major risk factor for post-transplant recurrence in children with SRNS, while transplant can be curative in genetic SRNS. A prolonged time spent on dialysis before transplantation has no protective effect on the risk of relapse and should not be encouraged. Retransplantation represents a second chance after graft loss for recurrence.
\end{abstract}

Keywords Steroid-resistant nephrotic syndrome $\cdot$ Kidney transplant $\cdot$ Post-transplant recurrence

Giovanni Montini

giovanni.montini@unimi.it

1 Pediatric Nephrology, Dialysis and Transplant Unit, Department of Clinical Sciences and Community Health, Fondazione IRCCS Ca' Granda-Ospedale Maggiore Policlinico, University of Milan, via della Commenda, 9, 20122 Milan, Italy

2 Division of Nephrology, Dialysis, and Transplantation, Scientific Institute for Research and Health Care, IRCCS Istituto Giannina Gaslini, Genoa, Italy

3 Renal Transplant Unit, Bambino Gesù Children's Research Hospital IRCCS, Rome, Italy
4 Pediatric Nephrology Unit, Regina Margherita Children's Hospital, Città della Salute e della Scienza di Torino, Turin, Italy

5 Pediatric Nephrology, Dialysis and Transplant Unit, Department of Women's and Children's Health, Hospital-University of Padova, Padua, Italy

6 North Italy Transplant program (NITp), UOC Coordinamento Trapianti, Fondazione IRCCS Ca' Granda-Ospedale Maggiore Policlinico, Milan, Italy 


\section{Introduction}

Steroid resistant nephrotic syndrome (SRNS) is the most common acquired cause of end stage renal failure (ESRD) requiring transplantation in children. Advances in genetic screening have allowed the identification of a monogenic cause of SRNS in one-third of cases [1]. Genetic SRNS are associated with an underlying mutations in genes encoding podocyte associated proteins, resulting in structural or functional disruption of the glomerular filtration barrier [2]. The pathophysiology of SRNS without underlying mutations remains poorly explained and is thought to involve an unknown circulating permeability factor [3] which may also be implicated in the recurrence soon after transplantation [4].

Unfortunately, in up to $50 \%$ of patients, SRNS relapses after transplantation and disease recurrence is a major cause of graft loss [4-6]. Genetic SRNS have been reported to have a low rate of recurrences [7-9]. On the other hand, previous studies have suggested non-African race, rapid progression to ESRD ( $<3$ years) and previous recurrence after transplantation to be associated with SRNS relapse $[8,10,11]$, but no established risk factors can actually predict the outcome. A longer time on dialysis before transplantation was believed to decrease the risk of relapse, but not confirmed by large reports $[12,13]$.

There is currently little consensus regarding the best management of post-transplant SRNS recurrence, which represents a devastating complication for families and physicians, and poses a significant threat to allograft survival. Plasma exchange (PE), steroids and rituximab are the most common strategies to treat the recurrence [14].

The objective of this study was to identify factors affecting the risk of recurrence and graft loss in children with SRNS, by stratifying the population according to their genetic status.

\section{Methods}

We performed a retrospective, multicentre, observational cohort study to address the long-term prognosis of renal graft, the risk factors for recurrence and the predictors for response to therapy following recurrence in paediatric patients undergoing renal transplantation because of a SRNS.

We identified patients who underwent renal transplantation at all five Italian paediatric transplant centres, between 2005 and 2017, with a primary diagnosis of SRNS and onset before 18 years. Patients were included if a clinical diagnosis of SRNS was made in an individual with otherwise unexplained nephrotic-range proteinuria refractory to standard steroid therapy and subsequently confirmed by renal biopsy showing a histological picture of focal segmental glomerulosclerosis (FSGS), minimal change disease (MCD), or diffuse mesangial sclerosis (DMS). Clinical records, pathology reports and genetic screening results were reviewed for the purposes of this study. Data were also collected about sex, age of disease onset, duration of pre-transplant dialysis, age at transplant, immunosuppression, allograft donor characteristics, disease recurrence, therapy for recurrence, and graft survival.

Patients were divided in three groups: Group A (Genetic SRNS): patients with an identified causative genetic mutation and/or a first degree relative with SRNS and/or extrarenal disease manifestations pathognomonic of SRNS, Group B (Idiopathic SRNS): patients with a negative or heterozygous recessive genetic test result and without a first degree family history or associated extra-renal manifestations pathognomonic of SRNS, Group C (unknown genetic status): patients with no genetic analysis performed and no family history or extra-renal manifestations typical of SRNS.

\section{Definitions}

Nephrotic-range proteinuria, urine protein:creatinine ratio $(\mathrm{uPr} / \mathrm{uCr}) \geq 2 \mathrm{mg} / \mathrm{mg}$. Age at disease onset, age at first clinical presentation of nephrotic syndrome. Steroid resistance, persistence of nephrotic range proteinuria following 4 weeks of daily $60 \mathrm{mg} / \mathrm{sqm}$ prednisone therapy. Post-transplant disease recurrence, an otherwise unexplained persistent nephrotic range proteinuria after renal transplantation, when rejection was excluded. Graft loss, functional failure of the renal allograft, necessitating renal replacement therapy. Remission after recurrence, complete resolution of proteinuria $(\mathrm{uPr} / \mathrm{uCr}<0.2 \mathrm{mg} / \mathrm{mg})$. Partial remission after recurrence, persistent reduction of proteinuria $(\mathrm{uPr} / \mathrm{uCr}<2 \mathrm{mg} /$ $\mathrm{mg}$ ) with preserved renal function.

\section{Statistical analysis}

Categorical variables were compared using the Chi squared test for independence. The distribution of continuous variables in groups was compared using the Wilcoxon signedrank test and the Kruskal-Wallis test. Linear regression models were used to compare continuous variables. For multivariate analysis, multiple logistic regression models were used. A $p$ value $<0.05$ was considered statistically significant. All statistical analyses were performed using the open source software R. (R Core Team, 2014. R: A language and environment for statistical computing, R Foundation for Statistical Computing, Vienna, Austria). 


\section{Results}

\section{Study cohort}

During the study period, a total of 728 (618 deceased and 110 living donors) renal grafts were performed at the 5 Italians pediatric transplant centres, of whom 123 in patients with ESRD secondary to SRNS. 101 patients received a first renal allograft and 22 a second renal transplant (12 failures of the original cohort and 10 failures of a first transplant that occurred before the study period). The number of patients who received a first transplant at each center is as follows: Istituto G. Gaslini, Genova-31, Bambino Gesù Children's Hospital, IRCCS, Rome-23, Fondazione IRCCS Ca' Granda, Ospedale Maggiore, Milan-20, Regina Margherita Children's Hospital, Turin-15, University Hospital of Padua-12. The study cohort is summarized in Fig. 1.

\section{First renal graft}

101 patients $(52.5 \%$ males) underwent a first renal transplant. The median age at onset was 2.8 years of age (range 0-17.2); 24 individuals (25.2\%) presented with congenital SRNS, defined as onset of disease within the first 3 months of life. Renal histology was consistent with FSGS in 85 cases, MCD in 14 and DMS in 2. Main demographic and clinical characteristics are summarized in Table 1. At transplant all patients received an induction therapy with basiliximab and immunosuppression with steroids, calcineurin inhibitors and mofetil mycophenolate. Two patients were treated with plasmapheresis pre-transplantation.

Genetic testing results were available for 76 individuals (75.2\%) (Table 2): 39 had an autosomal dominant mutation or were homozygous for a recessive mutation, 8 were heterozygous carriers and 29 had a negative genetic test. Genetic results were unavailable for 25 patients, among whom we were able to identify 2 additional patients with genetic SRNS: one had a sibling with established genetic SRNS and another showed extra-renal disease manifestations

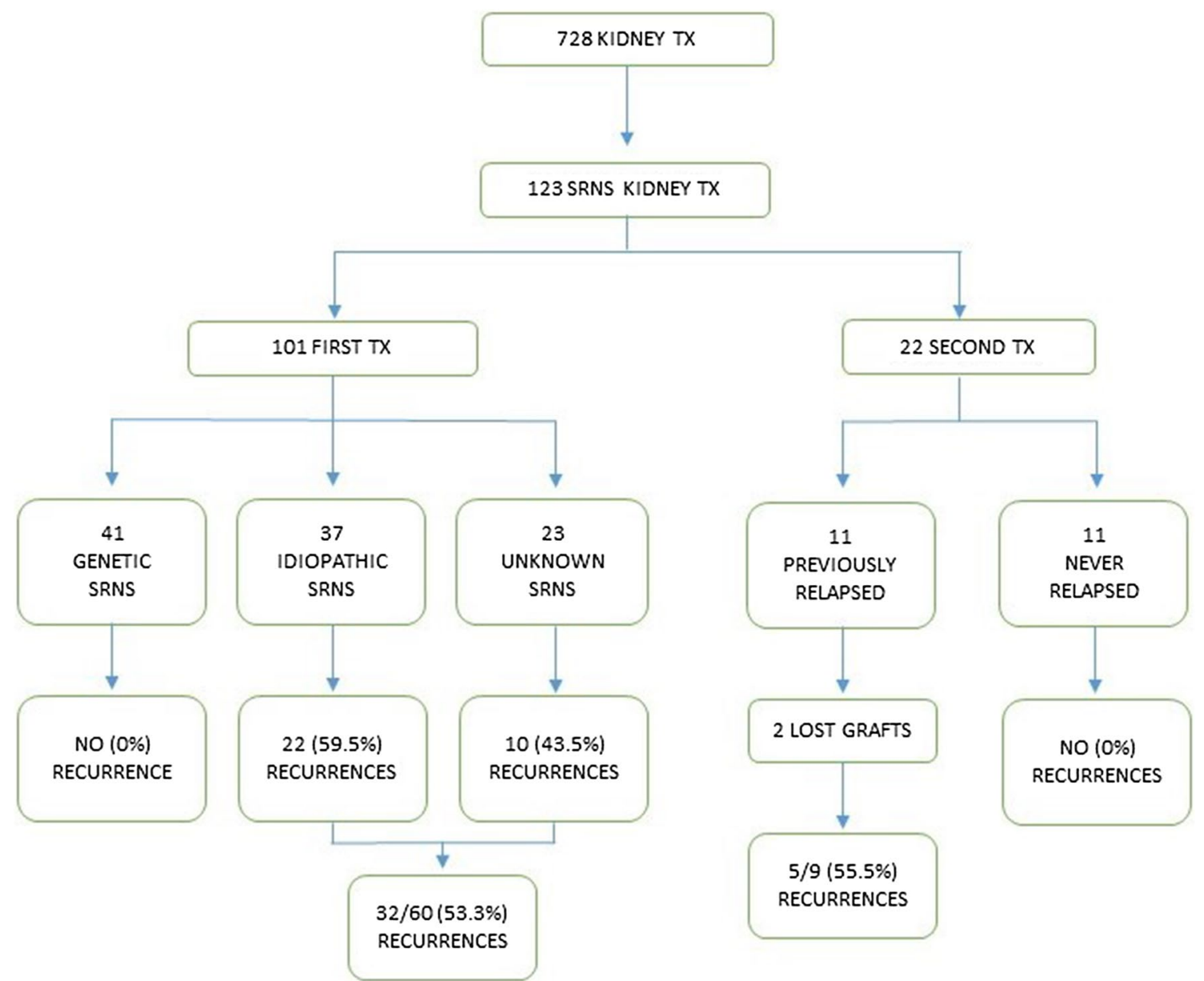

Fig. 1 Study cohort 
Table 1 Main demographic and clinical characteristics of SRNS children transplanted between 2005 and 2017

\begin{tabular}{lll}
\hline Characteristics & First renal Tx & Second renal Tx \\
\hline Total & 101 & 22 \\
Gender & & \\
Male & $53(52.5 \%)$ & $13(59.1 \%)$ \\
Female & $48(47.5 \%)$ & $9(40.9 \%)$ \\
Genetic disease & & \\
Yes & $41(40.6 \%)$ & $5(22.7 \%)$ \\
No & $37(36.6)$ & $13(56.6 \%)$ \\
Unknown & $23(22.8 \%)$ & $4(18.2 \%)$ \\
Age at onset (years) median (range) & $2.8(0-17.2)$ & $4.45(0-14.29)$ \\
Age at transplant (years) median (range) & $11.8(2.6-20.8)$ & $16.71(4.56-31.1)$ \\
Time to ESRD (years) median (range) & $3.3(1.7-14.3)$ & $2.5(0-7.5)$ \\
Time on dialysis before transplantation (years) median & $2(0-9)$ & Not available \\
(range) & & \\
Donor type & & $2(9.1 \%)$ \\
Living & $6(5.9 \%)$ & $20(90.9 \%)$ \\
Deceased & $95(94.1 \%)$ & $40(0-148)$ \\
Follow-up (months) median (range) & $58.5(0.7-157.8)$ &
\end{tabular}

Table 2 Prevalence of mutations among patients with available genetic results

\begin{tabular}{|c|c|c|c|}
\hline Gene & Encoded protein & Mode of inheritance & $\begin{array}{l}\text { Genetic } \\
\text { tests } \\
\mathrm{n}=76\end{array}$ \\
\hline NPHS1 & Nephrin & Recessive & 13 \\
\hline WT1 & Wilms tumour protein & Dominant & 11 \\
\hline NPHS2 & Podocin & Recessive & 8 \\
\hline ACTN4 & $\alpha$-Actinin & Dominant & 2 \\
\hline PLCE1 & Phospholipase C & Recessive & 1 \\
\hline COL4A5 & Type IV collagen $\alpha 5$ chain & $\mathrm{X}$-linked recessive & 1 \\
\hline SMARCAL1 & $\begin{array}{l}\text { SWI/SNF-related, matrix-associated, actin-dependent regulator } \\
\text { of chromatin, subfamily A-like protein } 1\end{array}$ & Recessive & 1 \\
\hline LMX1B & LIM homeobox transcription factor $1 \beta$ & Dominant & 1 \\
\hline COQ2 & Coenzyme Q2 & Recessive & 1 \\
\hline Heterozygous carriers & & Recessive & 8 \\
\hline No mutations & & & 29 \\
\hline
\end{tabular}

suggesting a genetic disease. The patient cohort was therefore comprised of: Group A (genetic SRNS): 41 individuals (40.6\%), Group B (idiopathic SRNS): 37 individuals (36.6\%), and Group C (unknown genetic status): 23 individuals (22.8\%). Age at the onset was similar between Group $\mathrm{B}$ (idiopathic) and Group C (unknown), while it was younger for patients with a genetic disease (Group A) $(p<0.0001)$.

NPHS1, encoding nephrin, was the single most commonly mutated gene and accounted for one-third (33.3\%) of positive genetic results, followed by WT1, encoding Wilms tumour protein and NPHS2, encoding podocin. Mutations in these genes were responsible of $28.2 \%$ and $20.5 \%$ of genetic SRNS, respectively. Taken together, mutations in NPHS1, NPHS2, and WT1 accounted for $82 \%$ of identified genetic cases. Pathogenetic mutations were also identified in the following genes: PLCE1, ACTN4, COL4A5, SMARCAL1, LMX1B, COQ2 (Table 2). In eight cases renal disease was associated with a syndromic presentation, as follows: Denys Drash syndrome in four cases and one case each of Frasier syndrome, WAGR syndrome, Leopard syndrome and Schimke immuno-osseous dysplasia.

\section{Post-transplant disease recurrence}

Median follow-up is 58.5 months (range 0.7-157.8). SRNS recurred in 32 individuals $(31.7 \%$ ) after the first renal transplant, at a median time of 2 days post-transplantation. When stratified by genetic status, the incidence of post-transplant 
disease recurrence was 59.5\% in Group B (idiopathic SRNS) and $43.5 \%$ in Group C (unknown genetic status). No Group A (genetic SRNS) child experienced disease recurrence and this group was therefore excluded from further analysis (Table 3).

The difference in post-transplant disease recurrence between Group B and C, however, was not significant $(p=0.23)$. Risk factors for recurrence were evaluated in the remaining 60 patients (Group B and C). Overall, SRNS recurred in 32/60 (53.3\%) non-genetic patients.

As all relapses except one (identified 10 years after transplantation) occurred within 8 months from transplant, the analysis was made at 8 months of follow-up and included all evaluable patients (54 patients). Age at transplant was categorized as $\geq 9$ years, following a ROC analysis identifying it as the best cut-off for relapse prediction in our dataset (Fig. 2). Bivariate analysis was performed by Wilcoxon test for independent samples. Multivariate analysis was performed by a logistic regression model.

At bivariate analysis, the following variable were significantly associated to relapse:

- Age $\geq 9$ years $(p=0.01823)$

- At least one HLA AB match $(p=0.01752)$

- At least one HLA DR match $(\mathrm{p}=0.01763)$

Gender, donor age and donor type (living or deceased) did not affect the risk of recurrence. Time to ESRD and duration of dialysis before transplant were not significantly associated with relapse; anyway, they were both longer in children with relapse (median $=4.6$ vs 2.7 years, $p=0.2673$ and 2.4 vs 1.8 years, $p=0.06582$, respectively).

We were not able to assess the role of different induction schedules, since all patients were homogeneously treated with basiliximab and immunosuppressive therapy. However, among the two patients treated with pre-transplantation PE, one experienced relapse the day after transplant.

Multivariate analysis included all the variables associated with recurrence at the bivariate analysis with a $\mathrm{p}$ value $<0.1$ (Table 4). Age at transplant $>9$ years and HLA-AB match were the only independent risk factors for recurrence after transplant ( $\mathrm{p}=0.01017$ and $\mathrm{p}=0.02465$, respectively). However, the best prediction model for relapse, characterized by

Table 3 Incidence of recurrence, stratified by genetic testing results

\begin{tabular}{llll}
\hline Characteristics & Total, $\mathrm{n}=101$ & $\begin{array}{l}\text { Recurrence, } \\
\mathrm{n}=32\end{array}$ & $\begin{array}{l}\text { No recurrence, } \\
\mathrm{n}=69\end{array}$ \\
\hline Genetic results & $n, \%$ & & \\
Negative & 37 & $22(59.5)$ & $15(40.5)$ \\
Unknown & 23 & $10(43.5)$ & $13(57.5)$ \\
Positive & 41 & $0(0.0)$ & $41(100.0)$ \\
\hline
\end{tabular}

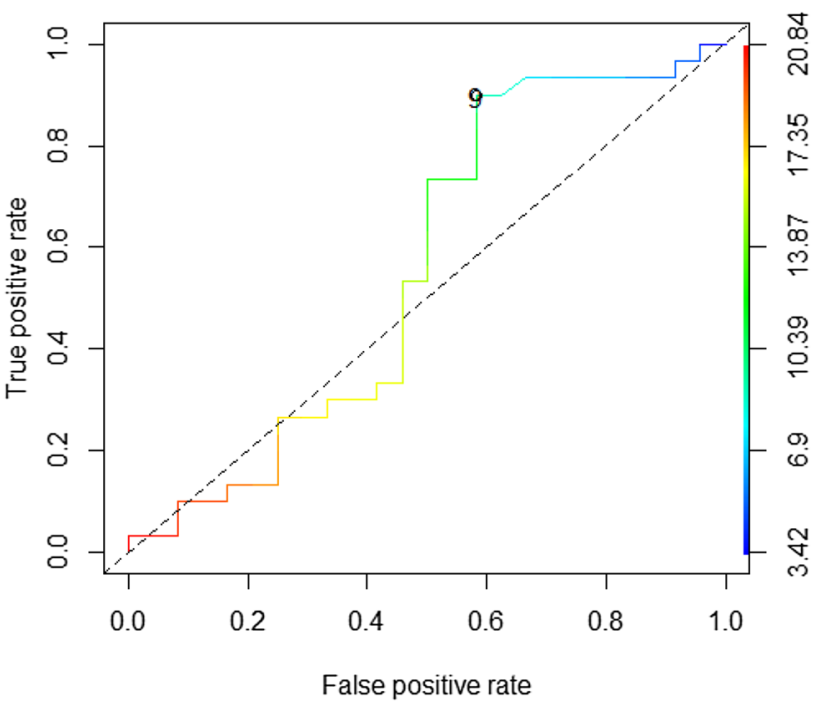

Fig. 2 ROC curve identifying the best cut-off for age at transplant with $\mathrm{FPR}=0.58333333$, TPR (sensitivity) $=0.86666667$, Specificity $=0.41666667, \mathrm{p}$ value $=0.01823$

the lowest residual deviance and lowest AIC, included also a longer duration of dialysis before transplant (Null deviance: 68.029 on 49 degrees of freedom; Residual deviance: 49.584 on 46 degrees of freedom; AIC: $57.584 ; p=0.06994)$. The model including the aforementioned 3 variables has a likelihood ratio test $p$ value of 0.000356 , and a pseudo $R$ squared value of 0.271136 (McFadden method).

The risk factors for recurrence in Group B and C are summarized in Table 5.

Disease recurrence was treated in all patients with PE with a median of 20 sessions (range 4-79). 22 were treated with rituximab and 9 with high dose steroids. The use of other therapeutic agents was as follows: ofatumumab (3), mesenchymal stromal cells (2), intravenous immunoglobulins (2), abatacept (2), cyclophosphamide (2), cyclosporin (1) and thymoglobulin (1). Overall, a complete or partial remission was achieved in 15 and 4 patients, respectively, 13 patients $(40.6 \%)$ failed to achieve sustained disease remission, despite treatment and 11/13 subsequently lost the graft. Use of rituximab or high dose steroids did not influence the response rate $(p=0.3574)$. The remission was persistent with preserved renal function in $13 / 15$ patients. One patient

Table 4 Variables included in the multivariate analysis

Variables

Age $\geq 9$ years

At least one HLA AB match

At least one HLA DR match

Duration of dialysis before transplant 
Table 5 Risk factors for post-transplant disease recurrence in Group B (idiopathic SRNS) and Group C (unknown genetic status) individuals, at 8 months of follow-up

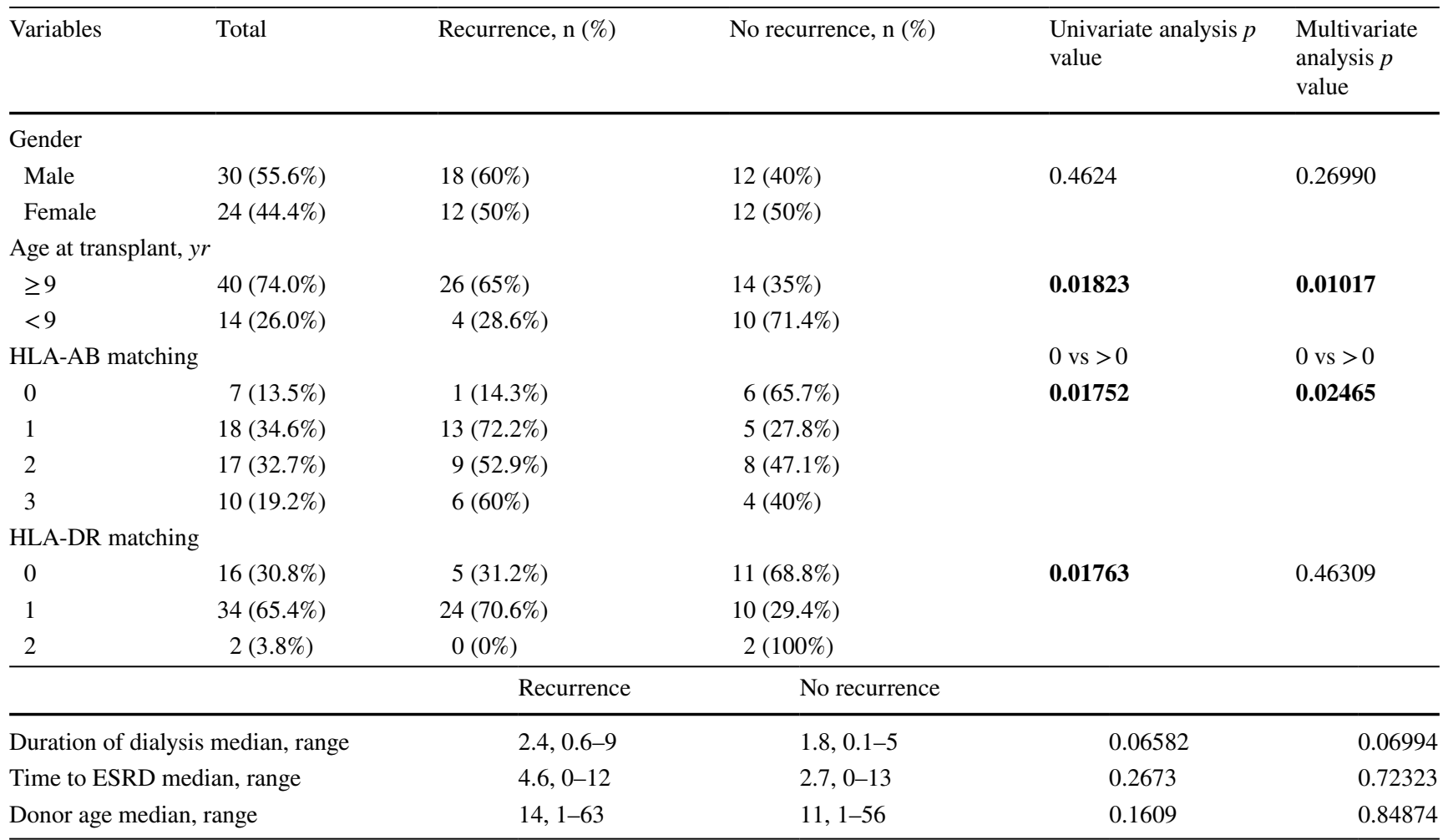

Statistically significant values in bold

had second untreatable relapse 10 years after transplant and lost the kidney. One additional patient with partial remission, following experienced rejection and lost his graft.

\section{Graft loss}

24 patients (23.8\%) experienced loss of a first renal graft. The causes of graft loss were as follows: disease recurrence in $12(50 \%)$, rejection in $6(25 \%)$, primary non-functioning graft in $3(12.5 \%)$, thrombosis in $2(8.3 \%)$ and chemotherapy toxicity for post-transplant thrombo-proliferative disease in one case (4.1\%). In addition, death with a functional graft due to sepsis occurred in two patients.

\section{Second renal transplant}

During the study period, 22 SRNS patients received a second renal graft; 11 of them had had a recurrence in the first graft, while 11 lost their transplant for different reasons. Among patients with a previous recurrence, 2/11 patients lost their graft immediately after the transplantation for reasons different from relapse (death and surgery complications) and were not included in the following analysis. After a median follow-up of 40 months, five patients relapsed on the second transplant. All of them have had a recurrence in the first graft. Therefore, in our population, only 5 out $9(55.5 \%)$ evaluable patients with a previous relapse experienced recurrence of proteinuria after the second kidney transplantation. Among five relapsed patients, only two subsequently lost the second graft. None of the 11 patients who lost the first transplant for different reasons suffered of relapse, with 4/11 having a genetic disease. Outside SRNS recurrence, two patients experienced a graft rejection and lost the second kidney graft and one patient died after a post-transplant lymphoproliferative disease.

\section{Discussion}

SRNS is a leading cause of ESRD in children. Post-transplant recurrence is a common complication, associated with an increased risk of graft loss. Many efforts have been made to identify the risk factors for recurrence in order to improve prevention and treatment strategies $[5,6,15]$.

Our study gives a clear picture of the Italian experience with kidney transplantation in children with SRNS, during a period of over 10 years, encompassing all the recent acquisition regarding the etiopathology and therapeutic options for SRNS. 
The overall incidence of post-transplant disease recurrence (53\% of non-genetic patients) is consistent with the available scientific literature, stretching back almost three decades $[16,17]$. Recurrence is confirmed to be a very early event, with a median time from transplant of 2 days and $30 / 32$ events occurring within the first 2 months after transplantation. By stratifying the cohort according to genetic status, we have been able to confirm that genetic SRNS does not recur after transplant. While previous reports have identified the genetic status as an important risk factor for disease recurrence, most available retrospective studies are unable to account for the genetic status of the majority of their cohorts [17, 18]. To the best of our knowledge, no previous studies were able to assess the risk of recurrence in an equally characterized population, as regards genetic disease. In our study, genetic results were indeed available for $76 / 101$ patients $(75.2 \%)$, furthermore since the remaining individuals (Group C, unknown genetic status) closely resemble Group B (Idiopathic SRNS) in key clinical features, including similar age of onset and rate of recurrence (43.5\%), it is likely that most of them also represent cases of idiopathic SRNS. Indeed, we believe that patients with early onset or congenital SRNS were more likely to be tested for a genetic disease, while genetic testing was less performed in older children and adolescents with a clinical picture of idiopathic SRNS.

Our observation is in line with previous studies that report no or very low relapse rate after transplantation in children with genetic SRNS [9, 17, 19]. Few old reports have suggested a risk of relapse for genetic SRNS, but they are almost all related to NPHS2 mutation, including heterozygous individuals [20-23]. The causative role of the variants included in these reports should be reconsidered, as exquisitely suggested in a recent review by Bierzynska [15].

The rate of recurrence in idiopathic SRNS (Group B) was $59.5 \%$. The result is slightly superior than previously reported. When genetic patients are excluded, Ding [17] and Pelletier [19] found a relapse rate of $46.3 \%$ and $47 \%$, respectively. The lower recurrence rate described by these groups could be justified by the presence of unknown genetic SRNS patients. Indeed, when both Group B and C are considered, the overall rate of recurrence was $53.3 \%$ in our cohort. Therefore, our data underline the importance of a genetic evaluation for SRNS genes in order to plan transplantation, as it represents the principle risk factor for recurrence.

Aside from absence of a genetic aetiology, our study identified age at transplant greater than 9 years and HLA-AB match as independent risk factors for recurrence. The best prediction model for recurrence included also a longer duration of dialysis.

Nehus et al. reported a higher rate of recurrence in younger children, among a cohort of 327 patients, though genetic results were not reported for any participants [24].
No significant difference in relapse rate according to the age at transplant were detected by Tejani and Stablein [12] and in the more recent studies by Ding et al. and Pelletier et al. [17, 19]. Again, unavailability of genetic testing for the majority of their patients could justify the different findings.

HLA-AB match was independently related to recurrence in our cohort, in contrast HLA AB or DR match did not influence the risk of relapse in the study by Tejani et al. [12] and did not affect transplant outcome in adolescent with SRNS in a retrospective study of the NAPRTCS registry [25].

Following the evidence that a circulating factor is responsible for recurrence, it has been suggested that a prolonged dialysis prior to renal transplantation would have a protective effect as far as the risk of relapse is concerned. The results of our study do not support this hypothesis. Indeed, in our study cohort, a longer duration of dialysis was associated with an increased risk of relapse. Even if this variable did not reach significance, its inclusion identifies the best prediction model for recurrence $\left(\mathrm{R}^{2} 0.271136\right)$. Among the few studies which investigated the association between duration of dialysis and disease recurrence, no significant differences were found in a single centre experience of 43 patients by Senggutuvan [16]. In a larger cohort of 132 paediatric renal transplants, found no relationship between disease recurrence and duration of dialysis was found [12]. Hence, since no protective effect was proven by others and our data show a longer duration of dialysis in patients with recurrence, even if not statistically significant, it is not justified to prolong the duration of dialysis before transplantation in children with SRNS.

Whether donor type (living vs deceased) is significantly associated with disease recurrence remains controversial. Data from old registries [26, 27] found no increased recurrence rates according to the type of donors. Other studies have suggested living donor as an independent risk factor for recurrence [17, 28, 29]. Our study cohort included only six living donor recipients (5.9\%), reflecting the reluctance of paediatric nephrologists to use living donors in SRNS patients, due to the risk of recurrence and graft loss. On the other hand, since in our cohort no relapses occurred in genetic SRNS, another important clinical implication of our study is that living kidney donors can be safely used in genetic SRNS patients.

All patients from our cohort were treated with PE, following SRNS recurrence. Complete or partial remission was achieved in 19/32 (59.4\%) children, with a functioning graft after a median follow-up of 39.5 months. Similar rates of response were previously reported. Kashgary et al. in their meta-analysis identified a remission rate of $70.2 \%$ in children treated with PE [14]. A lower response rate was reported by Pelletier [19], but remission information was available only 
for 49/64 (77\%) relapsed patients and the detailed immunosuppressive strategy is missing. According to our results, $\mathrm{PE}$ is confirmed as an effective treatment for recurrence. Even the small numbers, rituximab and high dose steroids did not influence the response rate in our cohort.

On the other hand, disease recurrence was the leading cause of graft loss in non-genetic SRNS and the rate of graft loss after relapse $(34.3 \%)$ in our study is consistent with previous data [26].

Among the small number of retransplanted individuals included in our study, the overall incidence of relapse in a second renal graft after a first recurrence is not significantly different from the first transplant (55\%). In $4 / 9$ patients who experienced a relapse in their first transplant, proteinuria did not recur after the second transplantation. This contrasts with reported small cohorts in whom the incidence of recurrence approaches $100 \%$ once the first transplant was lost for recurrent SRNS [10, 12, 28, 30]. We are not able to identify the factors responsible of the different outcome, but according to our data retransplantation after relapse can be considered in children with SRNS.

\section{Conclusions}

Twelve years of the Italian experience with post-transplant SRNS recurrence allows us to reach different important conclusions. Firstly, the absence of underlying genetic mutations predicts a high risk of post-transplant recurrence, therefore genetic screening must be performed in all children with SRNS before transplantation in order to best plan their care in the post-transplant period. Age $>9$ years is an independent risk factor for recurrence, while a prolonged time spent on dialysis before transplantation has no protective effect on the risk of relapse and should not be encouraged. Living donor did not influence the risk of relapse and can be safely used in genetic SRNS patients. PE based treatment strategies are effective in the majority of relapsed patients. Finally, in those who experience graft loss, even for recurrence, it is appropriate to consider retransplantation, as it maybe curative in the long term.

Acknowledgements We thank "La Nuova Speranza onlus" foundation for the management support.

Author Contributions WM designed the study, collected clinical data, performed data analysis and interpretation, drafted the article, and approved the final version of the manuscript. SP collected clinical data, performed data analysis, drafted the article, and approved the final version of the manuscript. GP performed the statistical analysis and data interpretation, drafted the article and approved the final version of the manuscript. GMG concepted the study, critically revised the article, and approved the final version of the manuscript. LDS collected clinical data, critically revised the article, and approved the final version of the manuscript. LP collected clinical data, critically revised the article, and approved the final version of the manuscript. LM collected clinical data, critically revised the article, and approved the final version of the manuscript. MC collected clinical data, critically revised the article, and approved the final version of the manuscript. IG collected clinical data, critically revised the article, and approved the final version of the manuscript. EC collected clinical data, critically revised the article, and approved the final version of the manuscript. EB collected clinical data, critically revised the article, and approved the final version of the manuscript. ST collected clinical data and approved the final version of the manuscript. LG collected clinical data and approved the final version of the manuscript. GC collected clinical data and approved the final version of the manuscript. MC collected clinical data and approved the final version of the manuscript. RT collected clinical data and approved the final version of the manuscript. GM concepted and designed the study, performed data interpretation, critically revised the article, and approved the final version of the manuscript.

Funding This study received no specific fund from any public, commercial, or not-for-profit agency.

\section{Compliance with ethical standards}

Conflict of interest WM has received a speaker honorarium from Sanofi-Genzyme. No other conflict of interest to declare.

Ethical statement The study was approved by the institutional review board of Fondazione IRCCS Ca' Granda Ospedale Maggiore Policlinico, Milan.

Informed consent Informed consent was not required in view of the retrospective study design and the anonymity of the patient records reviewed.

Open Access This article is distributed under the terms of the Creative Commons Attribution 4.0 International License (http://creativeco mmons.org/licenses/by/4.0/), which permits unrestricted use, distribution, and reproduction in any medium, provided you give appropriate credit to the original author(s) and the source, provide a link to the Creative Commons license, and indicate if changes were made.

\section{References}

1. Sadowski CE, Lovric S, Ashraf S et al (2015) A single-gene cause in $29.5 \%$ of cases of steroid-resistant nephrotic syndrome. J Am Soc Nephrol 26:1279-1289. https://doi.org/10.1681/ASN.20140 50489

2. Becherucci F, Mazzinghi B, Provenzano A et al (2016) Lessons from genetics: is it time to revise the therapeutic approach to children with steroid-resistant nephrotic syndrome? J Nephrol 29:543-550. https://doi.org/10.1007/s40620-016-0315-4

3. Irginia V, Avin JS, Am R et al (1996) Circulating factor associated with increased glomerular permeability to albumin in recurrent focal segmental glomerulosclerosis. N Engl J Med 334:878-883. https://doi.org/10.1056/NEJM199604043341402

4. Dall'Amico R, Ghiggeri G, Carraro M et al (1999) Prediction and treatment of recurrent focal segmental glomerulosclerosis after renal transplantation in children. Am J Kidney Dis 34:1048-1055. https://doi.org/10.1016/S0272-6386(99)70010-7

5. Weber S, Tönshoff B (2005) Recurrence of focal-segmental glomerulosclerosis in children after renal transplantation: clinical 
and genetic aspects. Transplantation 80:128-134. https://doi. org/10.1097/01.tp.0000187110.25512.82

6. Mahesh S, Del Rio M, Feuerstein D et al (2008) Demographics and response to therapeutic plasma exchange in pediatric renal transplantation for focal glomerulosclerosis: a single center experience. Pediatr Transplant 12:682-688. https://doi.org/10.11 11/j.1399-3046.2007.00880.x

7. Jungraithmayr TC, Hofer K, Cochat $P$ et al (2011) Screening for NPHS2 mutations may help predict FSGS recurrence after transplantation. J Am Soc Nephrol 22:579-585. https://doi. org/10.1681/asn.2010010029

8. Vinai M, Waber P, Seikaly MG (2010) Recurrence of focal segmental glomerulosclerosis in renal allograft: an in-depth review. Pediatr Transplant 14:314-325. https://doi.org/10.111 1/j.1399-3046.2009.01261.x

9. Weber S, Gribouval O, Esquivel EL et al (2004) NPHS2 mutation analysis shows genetic heterogeneity of steroid-resistant nephrotic syndrome and low post-transplant recurrence. Kidney Int 66:571579. https://doi.org/10.1111/j.1523-1755.2004.00776.x

10. Fine RN (2007) Recurrence of nephrotic syndrome/focal segmental glomerulosclerosis following renal transplantation in children. Pediatr Nephrol 22:496-502. https://doi.org/10.1007/ s00467-006-0361-6

11. Ulinski T (2010) Recurrence of focal segmental glomerulosclerosis after kidney transplantation: strategies and outcome. Curr Opin Organ Transplant 15:628-632. https://doi.org/10.1097/ MOT.0b013e32833dee3a

12. Tejani A, Stablein D (1992) Recurrence of focal segmental glomerulosclerosis posttransplantation: a special report of the North American Pediatric Renal Transplant Cooperative Study. J Am Soc Neph 2:S258-S263. https://doi.org/10.1017/CBO9781107 415324.004

13. Donckerwolcke RA, Broyer M, Brunner FP et al (1983) Combined report on regular dialysis and transplantation of children in Europe, XI, 1981. Proc Eur Dial Transplant Assoc 19:61-91

14. Kashgary A, Sontrop JM, Li L et al (2016) The role of plasma exchange in treating post-transplant focal segmental glomerulosclerosis: a systematic review and meta-analysis of 77 case-reports and case-series. BMC Nephrol 17:1-8. https://doi.org/10.1186/ s12882-016-0322-7

15. Bierzynska A, Saleem MA (2018) Deriving and understanding the risk of post-transplant recurrence of nephrotic syndrome in the light of current molecular and genetic advances. Pediatr Nephrol 33:2027-2035. https://doi.org/10.1007/s00467-017-3793-2

16. Senggutuvan P, Cameron JS, Hartley RB et al (1990) Recurrence of focal segmental glomerulosclerosis in transplanted kidneys: analysis of incidence and risk factors in 59 allografts. Pediatr Nephrol 4:21-28

17. Ding WY, Koziell A, McCarthy HJ et al (2014) Initial steroid sensitivity in children with steroid-resistant nephrotic syndrome predicts post-transplant recurrence. J Am Soc Nephrol 25:13421348. https://doi.org/10.1681/asn.2013080852

18. Hubsch H, Montan B, Abitbol C et al (2005) Recurrent focal glomerulosclerosis in pediatric renal allografts: the Miami experience. Pediatr Nephrol 20:210-216. https://doi.org/10.1007/ s00467-004-1706-7

19. Pelletier JH, Kumar KR, Engen R et al (2018) Recurrence of nephrotic syndrome following kidney transplantation is associated with initial native kidney biopsy findings. Pediatr Nephrol 34:539. https://doi.org/10.1007/s00467-018-4103-3

20. Bertelli R, Ginevri F, Caridi G et al (2003) Recurrence of focal segmental glomerulosclerosis after renal transplantation in patients with mutations of podocin. Am J Kidney Dis 41:1314-1321

21. Billing H, Müller D, Ruf R et al (2004) NPHS2 mutation associated with recurrence of proteinuria after transplantation. Pediatr Nephrol 19:561-564. https://doi.org/10.1007/s00467-003-1408-6

22. Becker-Cohen R, Bruschi M, Rinat C et al (2007) Recurrent nephrotic syndrome in homozygous truncating NPHS2 mutation is not due to anti-podocin antibodies. Am J Transplant 7:256-260. https://doi.org/10.1111/j.1600-6143.2006.01605.x

23. Caridi G, Bertelli R, Perfumo F, Ghiggeri GM (2004) Heterozygous NPHS1 or NPHS2 mutations in responsive nephrotic syndrome and the multifactorial origin of proteinuria. Kidney Int 66:1715-1716. https://doi.org/10.1111/j.1523-1755.2004.938_9.x

24. Nehus EJ, Goebel JW, Succop PS, Abraham EC (2013) Focal segmental glomerulosclerosis in children. Transplant J 96:550-554. https://doi.org/10.1097/TP.0b013e31829c2431

25. Baum MA, Ho M, Stablein D et al (2002) Outcome of renal transplantation in adolescents with focal segmental glomerulosclerosis. Pediatr Transplant 6:488-492

26. Abbott KC, Sawyers ES, Oliver JD et al (2001) Graft loss due to recurrent focal segmental glomerulosclerosis in renal transplant recipients in the United States. Am J Kidney Dis 37:366-373. https://doi.org/10.1053/ajkd.2001.21311

27. Hariharan S, Adams MB, Brennan DC et al (1999) Recurrent and de novo glomerular disease after renal transplantation: a report from Renal Allograft Disease Registry (RADR). Transplantation 68:635-641

28. First MR (1995) Living-related donor transplants should be performed with caution in patients with focal segmental glomerulosclerosis. Pediatr Nephrol 9(Suppl):S40-S42

29. Francis A, Trnka P, McTaggart SJ (2016) Long-term outcome of kidney transplantation in recipients with focal segmental glomerulosclerosis. Clin J Am Soc Nephrol 11:2041-2046. https:// doi.org/10.2215/CJN.03060316

30. Striegel JE, Sibley RK, Fryd DS, Mauer SM (1986) Recurrence of focal segmental sclerosis in children following renal transplantation. Kidney Int Suppl 19:S44-S50

Publisher's Note Springer Nature remains neutral with regard to jurisdictional claims in published maps and institutional affiliations. 